- Nota -

\title{
¿ESTÁ APROBADO EL PLAN DE GESTIÓN DEL DISTRITO DE CUENCA FLUVIAL DE CATALUÑA?
}

\author{
ISABEL CARO-PATÓN CARMONA \\ Profesora titular de Derecho Administrativo \\ Universidad de Valladolid \\ Menendez \& Asociados, Abogados \\ icaro@der.uva.es
}

Recibido: 15 de abril de 2011 / Aceptado: 24 de mayo de 2011

\section{RESUMEN:}

Pese a su publicación, cabe preguntarse si el Plan de Gestión del Distrito de Cuenca Fluvial de Cataluña está vigente, pues aún falta la preceptiva aprobación por el Gobierno español. El análisis del fallo de la STC 31/2010 no arroja luz sobre este interrogante pues ha declarado la constitucionalidad de la competencia exclusiva de la Generalitat sobre la planificación hidrológica de cuencas internas.

\section{RESUM:}

Malgrat la seva publicació, cal preguntar-se si el Pla de Gestió del Districte de conca fluvial de Catalunya està vigent, ja que encara falta la preceptiva aprovació pel Govern espanyol. L'anàlisi de la decisió de la STC 31/2010 no aclareix aquest interrogant ja que ha declarat la constitucionalitat de la competència exclusiva de la Generalitat sobre la planificació hidrològica de conques internes.

\section{ABSTRACT:}

Despite its publication, it remains unclear whether the Management Plan of the River Basin District of Catalonia is in force, as it still lacks the required approval by the 
Spanish government. The analysis of the Constitutional Court's decision STC 31/2010 sheds no light on this question since it has declared the constitutionality of the exclusive competence of the Government on the hydrological planning of basins.

PALABRAS CLAVE: Distribución competencial - STC 31/2010 — planificación hidrológica - aguas.

PARAULES CLAU: Distribució competencial — STC 31/2010 - planificació hidrològica - aigües.

KEY WORDS: Distribution of powers - STC 31/2010 — hydrological planning water policy.

Sumario: I. Introducción. II. La aprobación del Plan hidrológico por la Generalitat de Catalunya. III. Origen del problema. IV. La sentencia del Estatuto y la planificación hidrológica. 1. Primer nivel o análisis en el que se parte exclusivamente del fallo de la Sentencia. 2. Segundo nivel o análisis en el que se tiene en cuenca toda la interpretación que contiene la Sentencia V. Una primera conclusión. VI. ¿Es el PGDCF impugnable ante la jurisdicción contencioso-administrativa?

\section{INTRODUCCIÓN}

La reciente jurisprudencia constitucional recaída en los recursos interpuestos contra el modo en que distintos estatutos de autonomía han tratado el tema de las aguas está plagada de equívocos y, por distintas razones, no está ayudando a resolver los conflictos territoriales que existen.

Falta aún por recaer la sentencia que resuelve el recurso interpuesto contra la reserva hídrica del Estatuto de Aragón. Pero ya se conocen las sentencias de los estatutos valenciano (STC 247/2007), catalán (las SSTC 31/2010 y 138/2010 son las que tratan del agua), andaluz y castellano-leonés (SSTC de 18 y 21 de marzo de 2011, respectivamente).

El objeto de estas páginas no es un análisis completo de estas sentencias, sino con mucha más modestia analizar la repercusión de las sentencias del Estatuto catalán en la competencia sobre planificación hidrológica. Más en concreto, trato de buscar (sin 
hallarla) una respuesta a la pregunta que le pone el título: ¿está aprobado el Plan de Gestión de Distrito de Cuenca?

Es un comentario muy técnico que, sin embargo, desvela las importantes consecuencias que para la seguridad jurídica empieza a tener esta jurisprudencia.

\section{LA APROBACIÓN DEL PLAN HIDROLÓGICO POR LA GENERALITAT DE CATALUNYA}

El Plan de Gestión del Distrito de Cuenca Fluvial de Cataluña (PGDCFC) se ha aprobado por Decreto 188/2010, de 23 de noviembre, y se publicó en el Diari Oficial de la Generalitat de Catalunya de 26 de noviembre de 2010 (esto es, dos días antes de que tuvieran lugar las elecciones autonómicas de 28 de noviembre).

Con estos dos datos - hay un plan aprobado y publicado - en principio no cabe cuestionarse su existencia. Sin embargo, la lectura de su artículo primero abre un serio interrogante, pues se dice que se aprueba el Plan "sin perjuicio de las actuaciones que corresponda realizar a la Administración general del Estado".

La fórmula utilizada es absolutamente extraña en la aprobación de textos normativos. Y francamente no es fácil concluir si significa que el Plan ya puede aplicarse o si, por el contrario, que aún falta su tramitación por el Estado. De acuerdo con la legislación estatal (texto refundido de la Ley de Aguas, aprobado por Real Decreto Legislativo $1 / 2001$, en adelante, TRLA), es indiscutible que, tratándose de un plan hidrológico de aguas gestionadas por la Comunidad Autónoma, dos son las actuaciones pendientes: el informe del Consejo Nacional del Agua (art. 20.1.b TRLA) y la adopción de un Real Decreto del Consejo de Ministros, con refrendo real (art. 40.6 TRLA).

Pero si nos atenemos a la normativa interna autonómica, en particular al Reglamento de la Planificación Hidrológica, aprobado por Decreto 380/2006, en Cataluña parece que ya hay plan de cuenca. Al menos esto es lo que ha entendido el Gobierno de la Generalitat, pues deja sin aplicación el plan hidrológico anterior (disposición final primera). Ahora bien, todavía no se ha derogado este plan, que fue aprobado por Real Decreto del Gobierno de la nación, y, además, se ha omitido en el PGDCFC la obligada disposición relativa a la entrada en vigor. 
Para cualquier jurista de formación clásica, que no se sepa si una norma está en vigor es un verdadero despropósito, que en este caso se explica por la indefinición en que la Sentencia del Tribunal Constitucional sobre el Estatuto (STC 31/2010, de 28 de junio) ha dejado el tema relativo a las competencias sobre el agua.

\section{ORIGEN DEL PROBLEMA}

El origen de esta problemática se encuentra en la aprobación del Estatuto de Autonomía de Cataluña por Ley Orgánica 6/2006, de 19 de julio, que en su artículo 117.1.b) atribuyó a la Generalitat la competencia exclusiva para la planificación de cuencas hidrológicas intracomunitarias o internas (cuencas que solo se encuentran en una comunidad autónoma). El artículo 117, titulado “Aguas y obras hidráulicas”, dice así:

"1. Corresponde a la Generalitat, en materia de aguas que pertenezcan a cuencas hidrográficas intracomunitarias, la competencia exclusiva, que incluye en todo caso:

a) La ordenación administrativa, la planificación y la gestión del agua superficial y subterránea, de los usos y de los aprovechamientos hidráulicos, así como de las obras hidráulicas que no estén calificadas de interés general.

b) La planificación y la adopción de medidas e instrumentos específicos de gestión y protección de los recursos hídricos y de los ecosistemas acuáticos y terrestres vinculados al agua.

$[\ldots]$

2. La Generalitat, en los términos establecidos en la legislación estatal, asume competencias ejecutivas sobre el dominio público hidráulico y las obras de interés general. En estos mismos términos le corresponde la participación en la planificación y la programación de las obras de interés general.

3. La Generalitat participa en la planificación hidrológica y en los órganos de gestión estatales de los recursos hídricos y de los aprovechamientos hidráulicos que pertenezcan a cuencas hidrográficas intercomunitarias. Corresponde a la Generalitat, dentro de su ámbito territorial, la competencia ejecutiva sobre:

a) La adopción de medidas adicionales de protección y saneamiento de los recursos hídricos y de los ecosistemas acuáticos. 
b) La ejecución y la explotación de las obras de titularidad estatal si se establece mediante convenio.

c) Las facultades de policía del dominio público hidráulico atribuidas por la legislación estatal.

4. La Generalitat debe emitir un informe preceptivo para cualquier propuesta de trasvase de cuencas que implique la modificación de los recursos hídricos de su ámbito territorial.

5. La Generalitat participa en la planificación hidrológica de los recursos hídricos y de los aprovechamientos hidráulicos que pasen o finalicen en Cataluña provenientes de territorios de fuera del ámbito estatal español, de acuerdo con los mecanismos que establece el Título $\mathrm{V}$ y participará en su ejecución en los términos previstos por la legislación estatal”.

Esta asunción supuso un cambio respecto a la situación anterior, caracterizada por la competencia de la Administración del Estado en materia de planificación hidrológica en cuencas internas (art. 40.6 TRLA). En el momento de la aprobación del Estatuto, se pudo entender que en este aspecto concreto el Estatuto desplazaba la legislación estatal de $_{\text {aguas }_{2}}$ que atribuía al Gobierno de la nación la competencia para aprobar también los planes hidrológicos de las cuencas internas. Esta posición fue la adoptada en la defensa jurídica del Estatuto por el ejecutivo catalán_(antecedentes 58c de la STC 31/2010 sobre el Estatuto), que indicaba además que existían otros mecanismos para respetar las competencias estatales.

"Que la Ley de Aguas de 1985 atribuyera al Estado la decisión final no representaría límite alguno para el Estatuto, que, integrado en el bloque de constitucionalidad, puede configurar de otro modo, desde la plena fidelidad a las directivas constitucionales, las facultades comunitarias sobre un objeto por completo intracomunitario. La atribución en exclusiva a la Generalitat de la competencia para la planificación en materia de aguas intracomunitarias respeta la competencia estatal sobre aguas intercomunitarias y no implica en absoluto que, en su ejercicio, la Generalitat no deba respetar lo establecido por el Estado en materia de planificación económica en uso de la habilitación conferida por el art. 149.1.13 $\mathrm{CE}$, ni comporta ningún impedimento para la virtualidad de los mecanismos de coordinación estatales. La calificación de la competencia como exclusiva sería, por otra parte, compatible con la existencia de mecanismos coordinadores que encuentran su origen en el art. 149.1.13 CE". 
Esta lectura, sin embargo, no era compartida por los recurrentes, que impugnaron el artículo 117.1.a) por infracción de la legislación estatal dictada en ejercicio de competencias estatales y aplicable en todo el territorio nacional (y cuya constitucionalidad, por adecuación al reparto competencial, fue avalada por la STC 227/1988).

Creo que es importante situar el problema, que no era tanto la compatibilidad del artículo 117.1a) con la Constitución sino con la legislación básica estatal y, en concreto, con el artículo 40.6 del TRLA.

La discusión que subyace en el fondo, y por encima de la planificación hidrológica, es la posición de los estatutos de autonomía en relación con las leyes básicas estatales. La prioridad de un estatuto frente a la legislación básica del Estado (obviamente, la Constitución no incluye la materia "planificación hidrológica" en la lista de competencias estatales) era la posición de importantes administrativistas (GARCÍA DE ENTERRÍA ${ }^{1}$ ) y constitucionalistas $\left(\right.$ VIVER $^{2}$ ), pero no era la posición unánime en la doctrina. Para otros (FERNÁNDEZ FARRERES ${ }^{3}$, FANLO LORAS ${ }^{4}$ ), la legislación básica habría de prevalecer frente a lo que pudiera decir cualquier estatuto, pues de otra manera las competencias estatales quedarían al arbitrio de lo que dijera cada Comunidad Autónoma, y serían, por lo tanto, distintas en todo el territorio nacional.

Al margen del debate doctrinal, durante el proceso de elaboración del Plan hidrológico catalán la prevalencia de los estatutos frente a la legislación básica era la doctrina del TC. Esta postura se encontraba en la STC 247/2007 del Estatuto de Autonomía de la Comunidad Valenciana. En esta sentencia, y precisamente para evitar el desapoderamiento de competencias del Estado o una diversidad ineficiente entre el nivel

${ }^{1}$ GARCÍA DE ENTERRÍA, E. y FERNÁNDEZ RODRÍGUEZ, T. R., Curso de Derecho administrativo

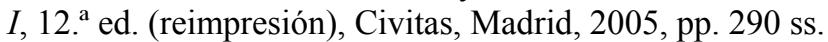

2 "Los efectos jurídicos de la sentencia sobre el Estatuto" en Revista Catalana de Dret Públic, número especial de la Sentencia sobre el Estatuto (s/n 2010), http://www10.gencat.net/eapc_revistadret?set_language=es\&cl=es.

${ }^{3}$ FERNÁNDEZ FARRERES, G., ¿Hacia una nueva doctrina constitucional del Estado autonómico? (Comentario a la STC 247/2007, de 12 de diciembre, sobre el Estatuto de Autonomía de la Comunidad Valenciana), Civitas, Madrid, 2009.

${ }^{4}$ FANLO LORAS, A. y GARRORENA MORALES, A., La Constitucionalidad de los nuevos estatutos en materia de aguas. A propósito de la propuesta de Estatuto de Autonomía de Castilla- La Mancha, Fundación Instituto Euromediterráneo del Agua, Murcia, 2008, p. 67. 
de competencias de cada Comunidad Autónoma, se exigía que la asunción estatutaria no vaciara de sentido la lista de competencias reservadas al Estado por la CE (art. 149.1), por lo que se establecieron unos límites que se entendieran bien. Esta doctrina constitucional implicaba, en sentido contrario, que si las asunciones competenciales de los estatutos invadían competencias del Estado, debían ser declaradas inconstitucionales.

Si el TC hubiera aceptado la doctrina de la STC 247/2007, el Plan hidrológico de cuencas internas estaría plenamente vigente porque cabría entender que el Estatuto desplazaba en el ámbito territorial de Cataluña el artículo 40.6 TRLA (y también el artículo 20 TRLA, que exige que haya un informe preceptivo del Consejo Nacional del Agua). Podía apoyar esta interpretación el hecho de que el Gobierno español no hubiera impugnado el Decreto catalán 380/2006 regulador de la planificación en cuencas internas, que ya reemplazaba la aprobación estatal del Plan por un informe preceptivo y determinante a emitir por el Estado en el procedimiento de su elaboración (art. 15.4, en su redacción dada por Decreto 31/2009, de 24 de febrero).

Sin embargo, como en la STC 31/2010 sobre el Estatuto el Tribunal Constitucional se ha apartado de la doctrina de la STC 247/2007 (sin justificar por qué ${ }^{5}$ ), el desconcierto generado es grande.

\footnotetext{
${ }^{5}$ En la STC 30/2011 (FJ 7..$^{\circ}$ ), que declara la inconstitucionalidad del Estatuto andaluz, se ha buscado la armonización de estas dos doctrinas: "En cuanto a las relaciones entre las normas estatutarias y la delimitación de las competencias del Estado ex art. 149.1 CE, hemos de partir de que si las primeras 'contribuye[n] a perfilar [...] el ámbito de normación y poder propio del Estado' ello es sólo 'en la medida en que las competencias del Estado dependen mediatamente en su contenido y alcance de la existencia y extensión de las competencias asumidas por las Comunidades Autónomas en el marco extraordinariamente flexible representado por el límite inferior o mínimo del art. $148 \mathrm{CE}$ y el máximo o superior, a contrario, del art. $149 \mathrm{CE}$. Esto no hace del Estatuto, sin embargo, una norma atributiva de las competencias del Estado' (STC 31/2010, de 28 de junio, FJ 5). El Estatuto de Autonomía tampoco es norma de ejercicio de las competencias estatales previstas por el art. 149.1 CE, de modo que, en caso de hacer alguna precisión sobre el alcance de éstas, su constitucionalidad pasa por el respeto de dos límites: el primero, de carácter positivo, consiste en que dicha precisión se 'haga para favorecer la mayor concreción de las competencias autonómicas que se correlacionan con' las competencias estatales que puedan presentar problemas de delimitación; y el segundo, de carácter negativo, reside en que la precisión estatutaria 'no impida el despliegue completo de las funciones propias de la competencia estatal regulada en el art. 149.1 CE de que se trate. Sólo si se satisfacen estas exigencias, tal modo de proceder resultará acorde a la Constitución' (STC 247/2007, FJ 10)".
} 


\section{LA SENTENCIA DEL ESTATUTO Y LA PLANIFICACIÓN HIDROLÓGICA}

El análisis de la Sentencia del Estatuto puede hacerse con distintos grados de profundidad.

\section{Primer nivel o análisis en el que se parte exclusivamente del fallo de la Sentencia}

El fallo de la Sentencia desestima expresamente la pretensión de que deba declararse inconstitucional el artículo 117.1.a) EA. Así, corresponde a la Comunidad Autónoma de Cataluña la competencia exclusiva sobre la planificación hidrológica. Si ceñimos el razonamiento o análisis al fallo de la Sentencia, el Plan de cuencas internas está vigente porque ha sido aprobado por la Administración competente.

\section{Segundo nivel o análisis en el que se tiene en cuenca toda la interpretación que contiene la Sentencia}

Pese a que no se haya anulado el artículo que atribuye a la Generalitat la competencia exclusiva en planificación hidrológica, en la fundamentación jurídica de la Sentencia se contiene una doctrina acabada en torno a la prevalencia de la legislación básica estatal sobre los textos estatutarios. Pero más aún, y en relación con el artículo 117.1 (y otros preceptos), indica que su constitucionalidad deriva de que todas las competencias ha de presumirse que se asumen bajo la cláusula "sin perjuicio de las del Estado". Es decir, aun cuando la norma no lo diga expresamente, cualquier operador jurídico ha de saber que las competencias autonómicas se subordinan a las del Estado, por más que en la materia concreta no se haga esta salvedad de forma expresa.

Así, en el FJ 64. ${ }^{\circ}$ de la STC 31/2010 se puede leer:

"En cuanto a la técnica seguida en ocasiones por el Estatuto de atribuir competencias materiales a la Generalitat que se proyectan «en todo caso» sobre las submaterias correspondientes, ya hemos afirmado (fundamento jurídico 59) que dicha expresión ha de entenderse en sentido meramente descriptivo o indicativo de que dichas submaterias forman parte del contenido de la realidad material de que se trate, pero sin que las competencias del Estado, tanto si son concurrentes como si son compartidas con las de la Comunidad Autónoma, resulten impedidas o limitadas en su ejercicio por esa atribución estatutaria «en todo caso» de 
competencias específicas a la Generalitat. Éste es el sentido en que habrá de ser entendida dicha expresión que figura en determinados preceptos impugnados (arts. $117.1 ; 118.1$ у $2 ; 120.1,2$ у $3 ; 121.1$ у $2 ; 123 ; 125.1$ у $4 ; 127.1$ у $2 ; 131.3 ; 132.1$; 133.1 у $4 ; 135.1 ; 139.1 ; 140.5$ у $7 ; 147.1 ; 149.3 ; 151 ; 152.4 ; 154.2 ; 155.1 ; 166.1,2$ y $3 ; 170.1$ y 172.2), lo que nos evitará volver sobre este extremo al enjuiciar cada uno de ellos".

Proyectando esta doctrina del texto de la Sentencia sobre la planificación hidrológica, el resultado es que la competencia exclusiva en planificación hidrológica corresponde en todo caso al Gobierno de la Generalitat, pero que esta exclusividad no impide el ejercicio de las competencias del Estado. Si se estuviera escribiendo esta nota sin pensar las palabras, la afirmación anterior podría quedarse en el papel sin plantear ningún problema de conciencia. Pero, puesto que estamos preguntándonos si el Plan hidrológico autonómico está aprobado, no cabe obviar la imposibilidad jurídica de que la Generalitat, al ejercer sus competencias exclusivas, apruebe el Plan sin que ello limite las competencias del Estado. Es decir, el ejercicio pleno de la competencia (aprobación del Plan por la Generalitat) es incompatible con la aplicación de la legislación básica (el Plan lo aprueba el Gobierno de la nación, previo informe del Consejo Nacional del Agua). Que no sepamos cuándo entra en vigor el Plan no es más que el primero de los signos de esta incompatibilidad.

El mérito del Gobierno de la Generalitat reside en el hecho de que, precisamente para respetar las competencias estatales, solicitó un informe preceptivo y determinante al Gobierno de la nación, informe que fue emitido en el procedimiento, como se desprende de la referencia específica que se hace en la exposición de motivos del Plan. Este informe era exigido por el Decreto 380/2006 (tras la reforma del Decreto 31/2009) precisamente como técnica interadministrativa para dar cabida a la intervención estatal considerada imprescindible incluso por el Gobierno catalán.

¿Por haberse solicitado este informe se están respetando las competencias estatales?

La respuesta es negativa si seguimos leyendo la STC, ya que esta señala en el FJ $65^{\circ}$ que:

"Ya hemos dicho y hemos de reiterar ahora que la atribución por el Estatuto de competencias exclusivas sobre una materia en los términos del art. 110 EAC no puede afectar a las competencias sobre materias o submaterias reservadas al Estado que se proyectarán, cuando corresponda, sobre las competencias exclusivas 
autonómicas con el alcance que les haya otorgado el legislador estatal con plena libertad de configuración, sin necesidad de que el Estatuto incluya cláusulas de salvaguarda de las competencias estatales (fundamentos jurídicos 59 y 64). Ello así, la competencia autonómica sobre cuencas hidrográficas intracomunitarias ha de cohonestarse con el legítimo ejercicio por parte del Estado de los títulos competenciales que puedan concurrir o proyectarse sobre dicha materia, en particular con el ejercicio de la competencia estatal sobre bases y coordinación de la planificación general de la actividad económica (art. 149.1.13 CE), que opera como límite a la competencia planificadora autonómica (STC 227/1988, de 29 de noviembre, FJ 20 a)".

Con este recordatorio, el TC no ha caído en la cuenta de que la intervención — recogida en el artículo 20 TRLA - del Consejo Nacional del Agua, que es un organismo para la gestión de las aguas, no parece encajar bien en el título económico que justifica la competencia del Estado. Pero este comentario presenta un problema menor si lo comparamos con el desconcierto que la aprobación del Plan produce.

Lo único que resulta evidente de la STC 31/2010 es que la competencia exclusiva atribuida por el Estatuto de Autonomía está completamente vacía de significado, ya que para el TC la delimitación competencial en esta materia deriva exclusivamente de la legislación estatal de aguas. En este sentido, la Sentencia 31/2010 viene a mantener que, al menos en lo que respecta a la atribución competencial, los estatutos de autonomía no son textos jurídicos, sino solo declaraciones de intenciones políticas cuya aplicación habrá de ser siempre contrastada con la legislación del Estado.

\section{UNA PRIMERA CONCLUSIÓN}

La conclusión es que para dar fuerza a la fundamentación jurídica de la Sentencia, el TC hubiera debido anular el artículo 117.1.a porque este problema práctico muestra que no es conciliable la competencia exclusiva en materia de planificación hidrológica con la aprobación de los planes de cuenca por el Estado. La no anulación del artículo 117.1.a genera una quiebra de la seguridad jurídica muy relevante para la marcha de la Administración hidráulica catalana y para el funcionamiento ordinario de la Administración pública en sus relaciones con los ciudadanos. 
$\mathrm{Y}$ es que al margen de todo lo anterior, el Plan puede aplicarse y producir efectos porque las normas jurídicas se presumen válidas desde su publicación, $\mathrm{y}$, por lo tanto, tienen plena eficacia desde que transcurre el plazo de vacatio legis.

\section{VI. ¿ES EL PGDCF IMPUGNABLE ANTE LA JURISDICCIÓN CONTENCIOSO-ADMINISTRATIVA?}

Se ha publicado en la prensa que el Gobierno de alguna Comunidad Autónoma limítrofe ha impugnado el PGDCF ante la jurisdicción contencioso-administrativa porque también planifica, en algunos aspectos, aguas de la cuenca del Ebro ${ }^{6}$. En concreto, el artículo 4.3 del PGDCF dispone que:

\section{"Las medidas que contiene este Plan que se refieren a la política de abastecimiento domiciliario y saneamiento, así como las relativas a la intervención administrativa en vertidos y gestión del medio, son aplicables en todo el territorio de Cataluña".}

En relación con este recurso se suscita otra cuestión de naturaleza jurídico-formal, que se responde de manera distinta en función de la perspectiva de análisis.

Pues, en efecto, desde la óptica de la legislación estatal de aguas, el Plan publicado en el boletín de la Generalitat es un acto de trámite (propuesta de plan), en mi opinión, inimpugnable porque no determina directa ni indirectamente el fondo del asunto (art. 25 de la Ley 29/1998). Esto es así porque el artículo 40 TRLA permite que el Estado ejerza un control de legalidad previo a la aprobación de los planes hidrológicos de cuencas internas. Entre los aspectos objeto de control de legalidad está precisamente comprobar que el Plan "no afecta a los recursos de otras cuencas" (art. 40 .6 TRLA). Podría ser que el Estado no llegara a aprobar el Plan si previamente no se eliminan los aspectos que puedan condicionar la planificación de la cuenca del Ebro (de momento, la única información disponible es que el Consejo Nacional del Agua, en su reunión de 24 de marzo de 2011, ha aprobado el informe presentado por el Estado en relación con este plan, pero no el contenido del informe). Y si esto fuera así, el recurso carecería de objeto, pero sencillamente ¡pero sencillamente por haberse presentado prematuramente!

\footnotetext{
6 Vid. Heraldo Aragón de de 04/02/2011 (http://www.heraldo.es/noticias/aragon_recurrido_plan_hidrologico_las_cuencas_internas_cataluna.html)
} 
A todo lo anterior, se tiene que añadir la doctrina de la STC 138/2010, que resuelve el recurso de inconstitucionalidad interpuesto por el Gobierno de La Rioja contra el Estatuto catalán. Entre otras cosas, La Rioja había impugnado el artículo $144.1 \mathrm{~g}$ ) del Estatuto, que se refiere a "la regulación y gestión de los vertidos efectuados en las aguas interiores de Cataluña, así como los efectuados en las aguas superficiales y subterráneas que no pasen por otra Comunidad Autónoma. En todo caso, dentro de su ámbito territorial, corresponde a la Generalitat la competencia ejecutiva sobre la intervención administrativa de los vertidos en las aguas superficiales y subterráneas". En el fundamento jurídico $4 .^{\circ}$ de esta sentencia se incardina la competencia en vertidos en materia de medio ambiente y se reconoce, por tanto, que las competencias de ejecución corresponden a la Comunidad Autónoma. Literalmente la STC 138/2010:

"El recurso alega, en segundo lugar, que la atribución a la Generalitat, dentro de su ámbito territorial, de la competencia ejecutiva sobre la intervención administrativa de los vertidos en las aguas superficiales y subterráneas -que incluyen los realizados en la cuenca del Ebro- es inconstitucional.

$\mathrm{Si}$, como hemos dicho, el encuadramiento adecuado de la materia "vertidos" es el título de medio ambiente, nada impide que el Estatuto de Autonomía de Cataluña incorpore tal atribución, pues el art. 149.1.23 CE reserva al Estado la «legislación básica», pudiendo las Comunidades asumir competencias ejecutivas, como así se hace [STC 149/1991, de 4 de julio, FF. 1 y 4 A)]”.

En consecuencia este motivo de impugnación debe rechazarse.

A la luz de esta sentencia, ¿es razonable que el Plan de gestión incluya los vertidos en el territorio catalán?, en otras palabras, ¿forma parte de la ejecución autonómica planificar los vertidos en su territorio?, ¿qué va a hacer el Estado en la aprobación del PGDCF? Lamentablemente, no me atrevo responder a estos interrogantes, porque tengo para mí que, bajo aguas tan turbias, los razonamientos jurídicos son de corto alcance. 